\title{
Towards Finite-Gap Integration of the Inozemtsev Model ${ }^{\star}$
}

\author{
Kouichi TAKEMURA
}

Department of Mathematical Sciences, Yokohama City University, 22-2 Seto, Kanazawa-ku, Yokohama 236-0027, Japan

E-mail: takemura@yokohama-cu.ac.jp

Received October 31, 2006, in final form February 07, 2007; Published online March 02, 2007

Original article is available at http://www.emis.de/journals/SIGMA/2007/038/

\begin{abstract}
The Inozemtsev model is considered to be a multivaluable generalization of Heun's equation. We review results on Heun's equation, the elliptic Calogero-Moser-Sutherland model and the Inozemtsev model, and discuss some approaches to the finite-gap integration for multivariable models.
\end{abstract}

Key words: finite-gap integration; Inozemtsev model; Heun's equation; Darboux transformation

2000 Mathematics Subject Classification: 81R12; 33E10; 34M35

\section{Introduction}

Differential equations defined on a complex domain frequently appear in mathematics and physics. One of the most important differential equations is the Gauss hypergeometric differential equation. Mathematically, it is a standard form of the second-order differential equation with three regular singularities on the Riemann sphere. Global properties of the solutions, i.e., the monodromy, often play decisive roles in applications to physics and mathematics.

A canonical form of a Fuchsian equation with four singularities is given by Heun's equation which is written as

$$
\left(\left(\frac{d}{d w}\right)^{2}+\left(\frac{\gamma}{w}+\frac{\delta}{w-1}+\frac{\epsilon}{w-t}\right) \frac{d}{d w}+\frac{\alpha \beta w-q}{w(w-1)(w-t)}\right) \tilde{f}(w)=0
$$

with the condition $\gamma+\delta+\epsilon=\alpha+\beta+1$. This equation appears in several topics in physics, i.e., astrophysics, crystalline materials and so on (see [37] and references therein). Although the problem of describing the monodromy of Heun's equation is much more difficult than that of the hypergeometric equation, Heun's equation has been studied from several viewpoints. A method of finite-gap integration is available on a study of Heun's equation, and consequently we have some formulae on the monodromy.

If there exists an odd-order differential operator $A=(d / d x)^{2 g+1}+\sum_{j=0}^{2 g-1} b_{j}(x)(d / d x)^{2 g-1-j}$ such that $\left[A,-d^{2} / d x^{2}+q(x)\right]=0$, then $q(x)$ is called the algebro-geometric finite-gap potential. Note that the equation $\left[A,-d^{2} / d x^{2}+q(x)\right]=0$ is equivalent to the function $q(x)$ being a solution to a stationary higher-order $\mathrm{KdV}$ equation (see [10]). In our setting, finite-gap integration is a method for analysis of the operator $-d^{2} / d x^{2}+q(x)$ where $q(x)$ is an algebro-geometric finitegap potential. Originally, the finite-gap property is a notion related to spectra. Let $H$ be the

\footnotetext{
${ }^{\star}$ This paper is a contribution to the Vadim Kuznetsov Memorial Issue "Integrable Systems and Related Topics".
} The full collection is available at http://www.emis.de/journals/SIGMA/kuznetsov.html 
operator $-d^{2} / d x^{2}+q(x)$, and the set $\sigma_{b}(H)$ be defined as follows:

$$
E \in \sigma_{b}(H) \Leftrightarrow \text { Every solution to }(H-E) f(x)=0 \text { is bounded on } x \in \mathbb{R} \text {. }
$$

If the closure of the set $\sigma_{b}(H)$ can be written as

$$
\overline{\sigma_{b}(H)}=\left[E_{0}, E_{1}\right] \cup\left[E_{2}, E_{3}\right] \cup \cdots \cup\left[E_{2 g}, \infty\right),
$$

where $E_{0}<E_{1}<\cdots<E_{2 g}$, i.e., the number of bounded bands is finite, then $q(x)$ is called the finite-gap potential. It was established in the 1970s that, under the assumption that $q(x)$ is a periodic, smooth, real function, the potential $q(x)$ is finite-gap if and only if $q(x)$ is algebrogeometric finite-gap.

On the approach by the finite-gap integration for Heun's equation, it is essential to transform Heun's equation into a form with the elliptic function. The transformed equation is written as

$$
(H-E) f(x)=\left(-\frac{d^{2}}{d x^{2}}+\sum_{i=0}^{3} l_{i}\left(l_{i}+1\right) \wp\left(x+\omega_{i}\right)-E\right) f(x)=0,
$$

where $\wp(x)$ is the Weierstrass $\wp$-function with periods $\left(2 \omega_{1}, 2 \omega_{3}\right), \omega_{0}(=0), \omega_{1}, \omega_{2}\left(=-\omega_{1}-\omega_{3}\right)$, $\omega_{3}$ are half-periods, and $l_{i}(i=0,1,2,3)$ are coupling constants. Here the variables $w$ and $x$ in equations (1.1), (1.2) are related by $w=\left(\wp(x)-\wp\left(\omega_{1}\right)\right) /\left(\wp\left(\omega_{2}\right)-\wp\left(\omega_{1}\right)\right)$. For details of the transformation, see $[34,38,43]$. The expression in terms of the elliptic function was already discovered in the 19th century, and some results which may relate to finite-gap integration were found in that era. For the case when three of $l_{0}, l_{1}, l_{2}, l_{3}$ are equal to zero, equation (1.2) is called Lamé's equation. Ince [19] established in 1940 that if $n \in \mathbb{Z}_{\geq 1}, \omega_{1} \in \mathbb{R} \backslash\{0\}$ and $\omega_{3} \in \sqrt{-1} \mathbb{R} \backslash\{0\}$, then the potential of Lamé's operator

$$
-\frac{d^{2}}{d x^{2}}+n(n+1) \wp\left(x+\omega_{3}\right)
$$

is finite-gap. In the late 1980s, Treibich and Verdier [48] found that the method of finite-gap integration is applicable for the case $l_{0}, l_{1}, l_{2}, l_{3} \in \mathbb{Z}$. Namely, they showed that the potential in equation (1.2) is an algebro-geometric finite-gap potential if $l_{i} \in \mathbb{Z}$ for all $i \in\{0,1,2,3\}$. Therefore the potential $\sum_{i=0}^{3} l_{i}\left(l_{i}+1\right) \wp\left(x+\omega_{i}\right)$ is called the Treibich-Verdier potential. Subsequently several others $[17,38,42,44,45,46]$ have produced more precise statements and concerned results on this subject. Namely, integral representations of solutions [38, 42], the Bethe Ansatz $[17,42]$, the global monodromy in terms of the hyperelliptic integrals [44], the Hermite-Krichever Ansatz [45] and a relationship with the Darboux transformation [46] were studied.

In this paper, we discuss some approaches to finite-gap integration for multivariable cases. A multivariable generalization of Heun's equation is given by the Inozemtsev model, which is a generalization of the Calogero-Moser-Sutherland model. The Inozemtsev model of type $B C_{N}$ [20] is a quantum mechanical system with $N$-particles whose Hamiltonian is given by

$$
\begin{aligned}
H= & -\sum_{j=1}^{N} \frac{\partial^{2}}{\partial x_{j}^{2}}+2 l(l+1) \sum_{1 \leq j<k \leq N}\left(\wp\left(x_{j}-x_{k}\right)+\wp\left(x_{j}+x_{k}\right)\right) \\
& +\sum_{j=1}^{N} \sum_{i=0}^{3} l_{i}\left(l_{i}+1\right) \wp\left(x_{j}+\omega_{i}\right),
\end{aligned}
$$

where $l$ and $l_{i}(i=0,1,2,3)$ are coupling constants. It is known that the Inozemtsev model of type $B C_{N}$ is completely integrable. More precisely, there exist operators of the form $H_{k}=$ 
$\sum_{j=1}^{N}\left(\frac{\partial}{\partial x_{j}}\right)^{2 k}+$ (lower terms) $(k=2, \ldots, N)$ such that $\left[H, H_{k}\right]=0$ and $\left[H_{k_{1}}, H_{k_{2}}\right]=0\left(k, k_{1}, k_{2}=\right.$

$2, \ldots, N)$. Note that the Inozemtsev model of type $B C_{N}$ is a universal completely integrable model of quantum mechanics with $B_{N}$ symmetry, which follows from the classification due to Ochiai, Oshima and Sekiguchi [30]. For the case $N=1$, the potential coincides with the Treibich-Verdier potential and the spectral problem for the Inozemtsev model of type $B C_{1}$ is equivalent to solving Heun's equation.

By the trigonometric limit $\left(\tau\left(=\omega_{3} / \omega_{1}\right) \rightarrow \sqrt{-1} \infty\right)$, we obtain the trigonometric CalogeroMoser-Sutherland model. The trigonometric model is well-studied by multivariable orthogonal polynomials (i.e., the Jack polynomial and the multivariable Jacobi polynomial). Vadim Kuznetsov and his collaborators studied multivariable orthogonal polynomials from the aspects of separation of variables [25], the Pfaff lattice [1] and the $Q$ operator [24]. Note that in the paper [26] relationships among separation of variables, integral transformations and Lamé's (Heun's) differential equation were discussed. Applications of these technique for models with elliptic potentials are anticipated.

Although the Inozemtsev model of type $B C_{N}$ is much more difficult than the trigonometric one, some approaches (perturbation from the trigonometric limit, quasi-solvability etc.) were introduced. Now we hope to develop analysis of this model by finite-gap integration. Although we can regard several works to be on this direction, in my opinion, they are still far from complete understanding of the model. We may consider the multivariable Darboux transformation as a possible approach, but we should develop it in the future.

This paper is organized as follows. In Section 2, we review the finite-gap integration of Heun's equation. An approach by the Darboux transformation is introduced. In Section 3, we collect results on the Calogero-Moser-Sutherland model and the Inozemtsev model. In Section 4, we discuss some approaches to finite-gap integration for those models.

\section{Finite-gap integration of Heun's equation}

\subsection{Darboux transformations and Heun's equation}

We consider the finite-gap property of Heun's equation in the elliptic form. It is known that the Treibich-Verdier potential is algebro-geometric finite-gap, i.e., there exists a differential operator $A$ of odd order which commutes with the operator $H^{\left(l_{0}, l_{1}, l_{2}, l_{3}\right)}$ where

$$
H^{\left(l_{0}, l_{1}, l_{2}, l_{3}\right)}=-\frac{d^{2}}{d x^{2}}+\sum_{i=0}^{3} l_{i}\left(l_{i}+1\right) \wp\left(x+\omega_{i}\right) .
$$

In this subsection, we construct an odd-order differential operator $A$ by composing the DarbouxCrum transformation which we will explain below.

We review the Darboux transformation. Let $\phi_{0}(x)$ be an eigenfunction of the operator $H=$ $-d^{2} / d x^{2}+q(x)$ corresponding to an eigenvalue $E_{0}$, i.e.

$$
\left(-\frac{d^{2}}{d x^{2}}+q(x)\right) \phi_{0}(x)=E_{0} \phi_{0}(x) .
$$

For this case, the potential $q(x)$ is written as $q(x)=\left(\phi_{0}^{\prime}(x) / \phi_{0}(x)\right)^{\prime}+\left(\phi_{0}^{\prime}(x) / \phi_{0}(x)\right)^{2}+E_{0}$. By setting $L=d / d x-\phi_{0}^{\prime}(x) / \phi_{0}(x)$ and $L^{\dagger}=-d / d x-\phi_{0}^{\prime}(x) / \phi_{0}(x)$, we have the factorization $H-E_{0}=L^{\dagger} L$. We set $\tilde{H}=L L^{\dagger}+E_{0}$. Then we have $\tilde{H}=-d^{2} / d x^{2}+q(x)-2\left(\phi_{0}^{\prime}(x) / \phi_{0}(x)\right)^{\prime}$ and the relation $\tilde{H} L=L H$. Hence, if $\phi(x)$ is an eigenfunction of the operator $H$ corresponding to the eigenvalue $E$, then $L \phi(x)$ is an eigenfunction of the operator $\tilde{H}$ corresponding to the eigenvalue $E$. This transformation is called the Darboux transformation. We generalize the operator $L$ to be the differential operator of higher order in the following proposition. 
Proposition 1 (cf. [3]). Suppose that the operator $H=-d^{2} / d x^{2}+q(x)$ preserves an $n$-dimensional space $U$ of functions. Let $L$ be the monic differential operator of order $n$ which annihilates all functions in $U$, and write

$$
L=\left(\frac{d}{d x}\right)^{n}+\sum_{i=1}^{n} c_{i}(x)\left(\frac{d}{d x}\right)^{n-i} .
$$

Set

$$
\tilde{H}=-d^{2} / d x^{2}+q(x)+2 c_{1}^{\prime}(x) .
$$

Then we have

$$
\tilde{H} L=L H .
$$

We call the operator $L$ in Proposition 1 the generalized Darboux transformation or the Darboux-Crum transformation. For the case $n=1$, let $\phi_{0}(x)$ be a non-zero function in $U$, then $U=\mathbb{C} \phi_{0}(x)$, the operator which annihilates $\phi_{0}(x)$ is given by $L=d / d x-\phi_{0}^{\prime}(x) / \phi_{0}(x)$ and the operator $\tilde{H}$ is given by $\tilde{H}=H-2\left(\phi_{0}^{\prime}(x) / \phi_{0}(x)\right)^{\prime}$. Hence the proposition reproduces the Darboux transformation for the case $n=1$.

We apply Proposition 1 to Heun's equation. For this purpose, we recall the quasi-solvability of Heun's equation. If a finite-dimensional space is invariant under an action of the Hamiltonian $H$ of a model, the model is partially solvable by diagonalizing the matrix representation of $H$. This situation is called quasi-solvable. On the quasi-solvability of Heun's equation, we have

Proposition 2 ([43], Proposition 5.1). Let $\alpha_{i}$ be a number such that $\alpha_{i}=-l_{i}$ or $\alpha_{i}=l_{i}+1$ for all $i \in\{0,1,2,3\}$, and set $d=-\sum_{i=0}^{3} \alpha_{i} / 2$. Suppose that $d \in \mathbb{Z}_{\geq 0}$, and let $V_{\alpha_{0}, \alpha_{1}, \alpha_{2}, \alpha_{3}}$ be the $d+1$-dimensional space spanned by

$$
\left\{\widehat{\Phi}(\wp(x)) \wp(x)^{n}\right\}_{n=0, \ldots, d},
$$

where $\widehat{\Phi}(z)=\left(z-e_{1}\right)^{\alpha_{1} / 2}\left(z-e_{2}\right)^{\alpha_{2} / 2}\left(z-e_{3}\right)^{\alpha_{3} / 2}$. Then the operator $H^{\left(l_{0}, l_{1}, l_{2}, l_{3}\right)}$ (see equation (2.1)) preserves the space $V_{\alpha_{0}, \alpha_{1}, \alpha_{2}, \alpha_{3}}$.

By applying Propositions 1 and 2, we obtain the following proposition after some calculations:

Proposition 3 ([46]). Let $\alpha_{i}$ be a number such that $\alpha_{i}=-l_{i}$ or $\alpha_{i}=l_{i}+1$ for all $i \in\{0,1,2,3\}$, and set $d=-\sum_{i=0}^{3} \alpha_{i} / 2$. Suppose that $d \in \mathbb{Z}_{\geq 0}$, and let $L_{\alpha_{0}, \alpha_{1}, \alpha_{2}, \alpha_{3}}$ be the monic differential operator of order $d+1$ which annihilates the space $V_{\alpha_{0}, \alpha_{1}, \alpha_{2}, \alpha_{3}}$. Then we have

$$
H^{\left(\alpha_{0}+d, \alpha_{1}+d, \alpha_{2}+d, \alpha_{3}+d\right)} L_{\alpha_{0}, \alpha_{1}, \alpha_{2}, \alpha_{3}}=L_{\alpha_{0}, \alpha_{1}, \alpha_{2}, \alpha_{3}} H^{\left(l_{0}, l_{1}, l_{2}, l_{3}\right)} .
$$

We construct an odd-degree differential operator $A$ which commutes with $H$ by composing the operators $L_{\alpha_{0}, \alpha_{1}, \alpha_{2}, \alpha_{3}}$. We define the operator $\tilde{L}_{\alpha_{0}, \alpha_{1}, \alpha_{2}, \alpha_{3}}$ as follows:

$$
\tilde{L}_{\alpha_{0}, \alpha_{1}, \alpha_{2}, \alpha_{3}}= \begin{cases}L_{\alpha_{0}, \alpha_{1}, \alpha_{2}, \alpha_{3}}, & \sum_{i=0}^{3} \alpha_{i} / 2 \in \mathbb{Z}_{\leq 0} \\ L_{1-\alpha_{0}, 1-\alpha_{1}, 1-\alpha_{2}, 1-\alpha_{3}}, & \sum_{i=0}^{3} \alpha_{i} / 2 \in \mathbb{Z}_{\geq 2} \\ 1, & \text { otherwise. }\end{cases}
$$


Set

$$
\begin{array}{llrl}
l_{0}^{e}=\left(-l_{0}+l_{1}+l_{2}+l_{3}\right) / 2, & l_{1}^{e}=\left(l_{0}-l_{1}+l_{2}+l_{3}\right) / 2, \\
l_{2}^{e}=\left(l_{0}+l_{1}-l_{2}+l_{3}\right) / 2, & l_{3}^{e}=\left(l_{0}+l_{1}+l_{2}-l_{3}\right) / 2, \\
l_{0}^{o}=\left(l_{0}+l_{1}+l_{2}+l_{3}+1\right) / 2, & l_{1}^{o}=\left(l_{0}+l_{1}-l_{2}-l_{3}-1\right) / 2, \\
l_{2}^{o}=\left(l_{0}-l_{1}+l_{2}-l_{3}-1\right) / 2, & l_{3}^{o}=\left(l_{0}-l_{1}-l_{2}+l_{3}-1\right) / 2 .
\end{array}
$$

The following proposition is proved by applying Proposition 3 four times:

Proposition 4 ([46]). Assume $l_{i} \in \mathbb{Z}_{\geq 0}$ for $i=0,1,2,3$. If $l_{0}+l_{1}+l_{2}+l_{3}$ is even, we set

$$
A=\tilde{L}_{-l_{3}^{e}, l_{2}^{e}+1, l_{1}^{e}+1,-l_{0}^{e}} \tilde{L}_{-l_{1}, l_{0}+1,-l_{3}, l_{2}+1} \tilde{L}_{-l_{0}^{e},-l_{1}^{e}, l_{2}^{e}+1, l_{3}^{e}+1} \tilde{L}_{-l_{0},-l_{1},-l_{2},-l_{3}},
$$

while if $l_{0}+l_{1}+l_{2}+l_{3}$ is odd, we set

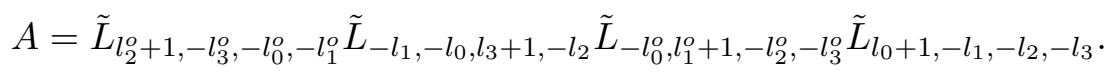

We then have that the operator $A$ commutes with $H^{\left(l_{0}, l_{1}, l_{2}, l_{3}\right)}$, i.e.,

$$
A H^{\left(l_{0}, l_{1}, l_{2}, l_{3}\right)}=H^{\left(l_{0}, l_{1}, l_{2}, l_{3}\right)} A .
$$

It is known that, if $l_{0}, l_{1}, l_{2}, l_{3} \in \mathbb{Z}_{\geq 0}$, then there exist four invariant spaces of the operator $H^{\left(l_{0}, l_{1}, l_{2}, l_{3}\right)}$, which we consider in Section 2.3, and the four operators in Proposition 4 are related to the four spaces.

Let $k_{i}$ be the rearrangement of $l_{i}$ such that $k_{0} \geq k_{1} \geq k_{2} \geq k_{3}(\geq 0)$. Set

$$
g=\left\{\begin{array}{lll}
k_{0}, & l_{0}+l_{1}+l_{2}+l_{3}: \text { even, } & k_{0}+k_{3} \geq k_{1}+k_{2} ; \\
\left(k_{0}+k_{1}+k_{2}-k_{3}\right) / 2, & l_{0}+l_{1}+l_{2}+l_{3}: \text { even, } & k_{0}+k_{3}<k_{1}+k_{2} ; \\
k_{0}, & l_{0}+l_{1}+l_{2}+l_{3}: \text { odd, } & k_{0} \geq k_{1}+k_{2}+k_{3}+1 \\
\left(k_{0}+k_{1}+k_{2}+k_{3}+1\right) / 2, & l_{0}+l_{1}+l_{2}+l_{3}: \text { odd, } & k_{0}<k_{1}+k_{2}+k_{3}+1
\end{array}\right.
$$

Then $g \in \mathbb{Z}_{\geq 0}$ and the degree of the operator $A$ is $2 g+1$.

For the case $l_{0}=2, l_{1}=l_{2}=l_{3}=0$, we have $g=2$ and the operator $A$ is expressed as

$$
\begin{aligned}
L_{2,-1,-1,0} L_{1,-2,1,0} L_{0,2,-1,-1} L_{-2,0,0,0} \\
=\left(\frac{d}{d x}+\frac{1}{2} \frac{\wp^{\prime}(x)}{\wp(x)-e_{1}}+\frac{1}{2} \frac{\wp^{\prime}(x)}{\wp(x)-e_{2}}\right)\left(\frac{d}{d x}+\frac{\wp^{\prime}(x)}{\wp(x)-e_{1}}-\frac{1}{2} \frac{\wp^{\prime}(x)}{\wp(x)-e_{2}}\right) \\
\times\left(\frac{d}{d x}-\frac{\wp^{\prime}(x)}{\wp(x)-e_{1}}+\frac{1}{2} \frac{\wp^{\prime}(x)}{\wp(x)-e_{2}}+\frac{1}{2} \frac{\wp^{\prime}(x)}{\wp(x)-e_{3}}\right) \\
\quad \times\left(\left(\frac{d}{d x}\right)^{2}-\frac{1}{2}\left(\frac{\wp^{\prime}(x)}{\wp(x)-e_{1}}+\frac{\wp^{\prime}(x)}{\wp(x)-e_{2}}+\frac{\wp^{\prime}(x)}{\wp(x)-e_{3}}\right) \frac{d}{d x}\right) \\
=\left(\frac{d}{d x}\right)^{5}-15 \wp(x)\left(\frac{d}{d x}\right)^{3}-\frac{45}{2} \wp^{\prime}(x)\left(\frac{d}{d x}\right)^{2}-9\left(5 \wp(x)^{2}-\frac{3}{4} g_{2}\right) \frac{d}{d x} .
\end{aligned}
$$

\subsection{Application of finite-gap property}

We investigate Heun's equation in the elliptic form

$$
(H-E) f(x)=0, \quad H=-\frac{d^{2}}{d x^{2}}+u(x), \quad u(x)=\sum_{i=0}^{3} l_{i}\left(l_{i}+1\right) \wp\left(x+\omega_{i}\right),
$$


by applying the finite-gap integration, which is based on the commutativity of $H\left(=-d^{2} / d x^{2}+\right.$ $u(x))$ and an odd-order differential operator $A$.

Since $A$ is a monic differential operator of order $2 g+1$, it can be expressed in the form

$$
A=(-1)^{g} \sum_{j=0}^{g}\left(\tilde{a}_{j}(x) \frac{d}{d x}+\tilde{b}_{j}(x)\right) H^{g-j},
$$

where $\tilde{a}_{0}(x)=1$. We have

$$
\begin{aligned}
0 & =\left[(-1)^{g} A, H\right]=\sum_{j=0}^{g}\left[\tilde{a}_{j}(x) \frac{d}{d x}+\tilde{b}_{j}(x),-\frac{d^{2}}{d x^{2}}+u(x)\right] H^{g-j} \\
& =\sum_{j=0}^{g}\left(\tilde{a}_{j}(x) u^{\prime}(x)+2 \tilde{a}_{j}^{\prime}(x) \frac{d^{2}}{d x^{2}}+\left(\tilde{a}_{j}^{\prime \prime}(x)+2 \tilde{b}_{j}^{\prime}(x)\right) \frac{d}{d x}+\tilde{b}_{j}^{\prime \prime}(x)\right) H^{g-j} \\
& =\sum_{j=0}^{g}\left(2 \tilde{a}_{j}^{\prime}(x)(-H+u(x))+\left(\tilde{a}_{j}^{\prime \prime}(x)+2 \tilde{b}_{j}^{\prime}(x)\right) \frac{d}{d x}+\tilde{a}_{j}(x) u^{\prime}(x)+\tilde{b}_{j}^{\prime \prime}(x)\right) H^{g-j} \\
& =\sum_{j=0}^{g}\left(\left(\tilde{a}_{j}^{\prime \prime}(x)+2 \tilde{b}_{j}^{\prime}(x)\right) \frac{d}{d x}-2 \tilde{a}_{j+1}^{\prime}(x)+2 \tilde{a}_{j}^{\prime}(x) u(x)+\tilde{a}_{j}(x) u^{\prime}(x)+\tilde{b}_{j}^{\prime \prime}(x)\right) H^{g-j} .
\end{aligned}
$$

Hence we obtain

$$
\tilde{b}_{j}(x)=-\tilde{a}_{j}^{\prime}(x) / 2+c_{j}, \quad \tilde{a}_{j}^{\prime \prime \prime}(x)-4 u(x) \tilde{a}_{j}^{\prime}(x)+4 \tilde{a}_{j+1}^{\prime}(x)-2 u^{\prime}(x) \tilde{a}_{j}(x)=0
$$

for some constants $c_{j}(j=0, \ldots, g)$. Therefore we have

Proposition 5. Set $\tilde{a}_{0}(x)=1$ and $\tilde{a}_{g+1}(x)=0$. The operator $A$ may be expressed in the form

$$
A=(-1)^{g}\left[\sum_{j=0}^{g}\left\{\tilde{a}_{j}(x) \frac{d}{d x}-\frac{1}{2}\left(\frac{d}{d x} \tilde{a}_{j}(x)\right)\right\} H^{g-j}+\sum_{j=0}^{g} c_{j} H^{g-j}\right],
$$

for some functions $\tilde{a}_{j}(x)(j=1, \ldots, g)$ and constants $c_{j}(j=0, \ldots, g)$, where the functions $\tilde{a}_{j}(x)$ $(j=0, \ldots, g)$ satisfy

$$
\tilde{a}_{j}^{\prime \prime \prime}(x)-4 u(x) \tilde{a}_{j}^{\prime}(x)+4 \tilde{a}_{j+1}^{\prime}(x)-2 u^{\prime}(x) \tilde{a}_{j}(x)=0 .
$$

We define a function $\Xi(x, E)$ which plays the important role for the solutions and the monodromy of Heun's equation. Set

$$
\Xi(x, E)=\sum_{i=0}^{g} \tilde{a}_{g-i}(x) E^{i} .
$$

It follows from equation (2.6) that $\Xi(x, E)$ satisfies a differential equation satisfied by products of any pair of the solutions to equation (2.4), i.e.,

$$
\left(\frac{d^{3}}{d x^{3}}-4(u(x)-E) \frac{d}{d x}-2 u^{\prime}(x)\right) \Xi(x, E)=0 .
$$

On the basis of Proposition 5 and the function $\Xi(x, E)$ in equation $(2.7)$, we have 
Proposition 6 ([46]). (i) The constants $c_{j}(j=1, \ldots, g)$ in equation (2.5) are all zero.

(ii) The function $\Xi(x, E)$ is even doubly-periodic and expressed as

$$
\Xi(x, E)=c_{0}(E)+\sum_{i=0}^{3} \sum_{j=0}^{l_{i}-1} b_{j}^{(i)}(E) \wp\left(x+\omega_{i}\right)^{l_{i}-j},
$$

where the coefficients $c_{0}(E)$ and $b_{j}^{(i)}(E)$ are polynomials in $E$. The coefficients do not have common divisors and the polynomial $c_{0}(E)$ is monic. We have $g=\operatorname{deg}_{E} c_{0}(E)$ and the coefficients satisfy $\operatorname{deg}_{E} b_{j}^{(i)}(E)<g$ for all $i$ and $j$.

For the case $l_{0}=2, l_{1}=l_{2}=l_{3}=0$, we have

$$
\Xi(x, E)=E^{2}+3 E_{\wp}(x)+9 \wp(x)^{2}-\frac{9}{4} g_{2} .
$$

where $e_{i}=\wp\left(\omega_{i}\right)$ and $g_{2}=-4\left(e_{1} e_{2}+e_{2} e_{3}+e_{3} e_{1}\right)$.

Note that the function $\Xi(x, E)$ can be also obtained as the function satisfying equation $(2.8)$ and Proposition 6 (ii) (see [42]).

We can derive an integral formula for a solution to equation (2.4) in terms of the doubly periodic function $\Xi(x, E)$. Set

$$
\begin{aligned}
Q(E)= & \Xi(x, E)^{2}\left(E-\sum_{i=0}^{3} l_{i}\left(l_{i}+1\right) \wp\left(x+\omega_{i}\right)\right) \\
& +\frac{1}{2} \Xi(x, E) \frac{d^{2} \Xi(x, E)}{d x^{2}}-\frac{1}{4}\left(\frac{d \Xi(x, E)}{d x}\right)^{2} .
\end{aligned}
$$

It is shown by differentiating the right-hand side of equation (2.10) and applying equation (2.8) that $Q(E)$ is independent of $x$. Thus $Q(E)$ is a monic polynomial in $E$ of degree $2 g+1$, which follows from the expression for $\Xi(x, E)$ given by equation $(2.9)$. For the case $l_{0}=2$, $l_{1}=l_{2}=l_{3}=0$, we have

$$
Q(E)=\left(E^{2}-3 g_{2}\right) \prod_{i=1}^{3}\left(E-3 e_{i}\right) .
$$

The following proposition on the integral representation of a solution to equation (2.4) was obtained in [42]:

Proposition 7 ([42], Proposition 3.7). Let $\Xi(x, E)$ be the doubly periodic function defined in equation (2.7) and $Q(E)$ be the monic polynomial defined in equation (2.10). Then the function

$$
\Lambda(x, E)=\sqrt{\Xi(x, E)} \exp \int \frac{\sqrt{-Q(E)} d x}{\Xi(x, E)}
$$

is a solution to the differential equation (2.4).

If the value $E$ satisfies $Q(E) \neq 0$, then the functions $\Lambda(x, E)$ and $\Lambda(-x, E)$ form a basis of solutions to equation (2.4). Since equation (2.4) is doubly-periodic, the functions $\Lambda\left(x+2 \omega_{k}, E\right)$ and $\Lambda\left(-\left(x+2 \omega_{k}\right), E\right)$ are also solutions to equation (2.4). We consider the monodromy on the functions $\Lambda(x, E)$ and $\Lambda(-x, E)$. Note that, if $\Lambda\left(x+2 \omega_{k}, E\right)$ is expressed as $B(E) \Lambda(x, E)$, then $\Lambda\left(-\left(x+2 \omega_{k}\right), E\right)$ is expressed as $B(E)^{-1} \Lambda(-x, E)$. We will express $B(E)$ as a hyperelliptic integral of second kind. We rewrite the function $\Xi(x, E)$ and define $a(E)$ as follows:

$$
\Xi(x, E)=c(E)+\sum_{i=0}^{3} \sum_{j=0}^{l_{i}-1} a_{j}^{(i)}(E)\left(\frac{d}{d x}\right)^{2 j} \wp\left(x+\omega_{i}\right), \quad a(E)=\sum_{i=0}^{3} a_{0}^{(i)}(E) .
$$


Proposition 8 ([44], Theorem 3.7). Assume $l_{i} \in \mathbb{Z}_{\geq 0}(i=0,1,2,3)$. Let $E_{0}$ be a value such that $Q\left(E_{0}\right)=0$. Then $\Lambda\left(x+2 \omega_{k}, E_{0}\right)=(-1)^{q_{k}} \Lambda\left(x, E_{0}\right)$ for $q_{k} \in\{0,1\}(k=1,3)$ and we have

$$
\Lambda\left(x+2 \omega_{k}, E\right)=(-1)^{q_{k}} \Lambda(x, E) \exp \left(-\frac{1}{2} \int_{E_{0}}^{E} \frac{-2 \eta_{k} a(\tilde{E})+2 \omega_{k} c(\tilde{E})}{\sqrt{-Q(\tilde{E})}} d \tilde{E}\right),
$$

where $\eta_{k}=\zeta\left(\omega_{k}\right)(k=1,3)$ and $\zeta(x)$ is the Weierstrass zeta function.

For the case $l_{0}=2, l_{1}=l_{2}=l_{3}=0$, we set $E_{0}=\sqrt{3 g_{2}}$. Then $q_{1}=q_{3}=0$ and the function $a(E)$ and $c(E)$ are determined as

$$
c(E)=E^{2}-\frac{3}{2} g_{2}, \quad a_{0}(E)=3 E .
$$

Hence we have

$$
\Lambda\left(x+2 \omega_{k}, E\right)=\Lambda(x, E) \exp \left(-\frac{1}{2} \int_{\sqrt{3 g_{2}}}^{E} \frac{-6 \tilde{E} \eta_{k}+\left(2 \tilde{E}^{2}-3 g_{2}\right) \omega_{k}}{\sqrt{-\left(\tilde{E}^{2}-3 g_{2}\right) \prod_{i=1}^{3}\left(\tilde{E}-3 e_{i}\right)}} d \tilde{E}\right) .
$$

We review the propositions related with the Bethe Ansatz (Proposition 9) and the HermiteKrichever Ansatz (Proposition 10), which are also reductions of the finite-gap property.

Proposition 9 ([42], Theorem 3.12). (i) If the value $E$ satisfies $Q(E) \neq 0$, then there exists $t_{1}, \ldots, t_{n}$ and $C$ such that $t_{j} \neq t_{j^{\prime}}\left(j \neq j^{\prime}\right), t_{j} \notin \omega_{1} \mathbb{Z}+\omega_{3} \mathbb{Z}$ and $\Lambda(x, E)$ is expressed as

$$
\Lambda(x, E)=C \frac{\prod_{j=1}^{l} \sigma\left(x+t_{j}\right)}{\sigma(x)^{l_{0}} \sigma_{1}(x)^{l_{1}} \sigma_{2}(x)^{l_{2}} \sigma_{3}(x)^{l_{3}}} \exp \left(-x \sum_{i=1}^{l} \zeta\left(t_{j}\right)\right),
$$

where $\sigma(x)$ is the Weierstrass sigma function and $\sigma_{i}(x)(i=1,2,3)$ are the co-sigma functions defined by

$$
\sigma_{i}(z)=\exp \left(-\eta_{i} z\right) \sigma\left(z+\omega_{i}\right) / \sigma\left(\omega_{i}\right)
$$

(ii) The function

$$
\tilde{\Lambda}(x)=\frac{\prod_{j=1}^{l} \sigma\left(x+t_{j}\right)}{\sigma(x)^{l_{0}} \sigma_{1}(x)^{l_{1}} \sigma_{2}(x)^{l_{2}} \sigma_{3}(x)^{l_{3}}} \exp (c x),
$$

with the condition $t_{j} \neq t_{j^{\prime}}\left(j \neq j^{\prime}\right)$ and $t_{j} \notin \omega_{1} \mathbb{Z}+\omega_{3} \mathbb{Z}$ is an eigenfunction of the operator $H$ (see equation (2.4)), if and only if $t_{j}(j=1, \ldots, l)$ and $c$ satisfy the relations,

$$
\begin{aligned}
& \sum_{k \neq j} \zeta\left(-t_{j}+t_{k}\right)-l_{0} \zeta\left(-t_{j}\right)-\sum_{i=1}^{3} l_{i}\left(\zeta\left(-t_{j}+\omega_{i}\right)-\zeta\left(\omega_{i}\right)\right)=-c, \quad(j=1, \ldots, l), \\
& \left(1-\delta_{l_{0}, 0}\right)\left(c+\sum_{j=1}^{l} \zeta\left(t_{j}\right)\right)=0, \\
& \left(1-\delta_{l_{i}, 0}\right)\left(c+l \zeta\left(\omega_{i}\right)+\sum_{j=1}^{l} \zeta\left(-\omega_{i}+t_{j}\right)\right)=0, \quad(i=1,2,3) .
\end{aligned}
$$


The eigenvalue $E$ is given by

$$
\begin{aligned}
E= & -c^{2}+\left(l_{0} l_{1}+l_{2} l_{3}\right) e_{1}+\left(l_{0} l_{2}+l_{1} l_{3}\right) e_{2}+\left(l_{0} l_{3}+l_{1} l_{2}\right) e_{3}-\sum_{i=1}^{3} l_{i} \eta_{i}\left(2 c+l \eta_{i}\right) \\
& -\sum_{j=1}^{l} \sum_{i=0}^{3} l_{i}\left(\wp\left(t_{j}-\omega_{i}\right)-\zeta\left(t_{j}-\omega_{i}\right)^{2}\right)+\sum_{j<k}\left(\wp\left(t_{j}-t_{k}\right)-\zeta\left(t_{j}-t_{k}\right)^{2}\right) .
\end{aligned}
$$

Equation (2.13) is called the Bethe Ansatz equation for the Inozemtsev model of type $B C_{1}$ (see [42]). Note that Gesztesy and Weikard [17] obtained similar results. The monodromy of the function $\tilde{\Lambda}(x)$ in equation $(2.12)$ is written as

$$
\tilde{\Lambda}\left(x+2 \omega_{k}\right)=\exp \left(2 \eta_{k}\left(t_{1}+\cdots+t_{l}\right)+2 \omega_{k}\left(c-\zeta\left(t_{1}\right)-\cdots-\zeta\left(t_{l}\right)\right)\right) \tilde{\Lambda}(x)
$$

for $k=1,2,3$.

In order to describe the proposition on the Hermite-Krichever Ansatz, we define

$$
\Phi_{i}(x, \alpha)=\frac{\sigma\left(x+\omega_{i}-\alpha\right)}{\sigma\left(x+\omega_{i}\right)} \exp (\zeta(\alpha) x), \quad(i=0,1,2,3)
$$

Proposition 10 ([45]). There exist polynomials $P_{1}(E), \ldots, P_{6}(E)$ such that, if $P_{2}(E) \neq 0$, then $\Lambda(x, E)$ is written as

$$
\Lambda(x, E)=\exp (\kappa x)\left(\sum_{i=0}^{3} \sum_{j=0}^{l_{i}-1} \tilde{b}_{j}^{(i)}\left(\frac{d}{d x}\right)^{j} \Phi_{i}(x, \alpha)\right)
$$

for some values $\tilde{b}_{j}^{(i)}\left(i=0, \ldots, 3, j=0, \ldots, l_{i}-1\right), \alpha$ and $\kappa$. The values $\alpha$ and $\kappa$ are expressed as

$$
\wp(\alpha)=\frac{P_{1}(E)}{P_{2}(E)}, \quad \wp^{\prime}(\alpha)=\frac{P_{3}(E)}{P_{4}(E)} \sqrt{-Q(E)}, \quad \kappa=\frac{P_{5}(E)}{P_{6}(E)} \sqrt{-Q(E)} .
$$

For the periodicity of the function $\Lambda(x, E)$, we have

$$
\Lambda\left(x+2 \omega_{k}, E\right)=\exp \left(-2 \eta_{k} \alpha+2 \omega_{k} \zeta(\alpha)+2 \kappa \omega_{k}\right) \Lambda(x, E)
$$

for $k=1,3$.

Note that $\alpha$ in equation (2.14) and $t_{j}$ in equation (2.11) satisfy the relation $\alpha=-\sum_{j=1}^{l} t_{j}$. To calculate the polynomials $P_{1}(E), \ldots, P_{6}(E)$, it is effective to apply the notions "twisted Heun polynomial" and "theta-twisted Heun polynomial" (see [45]).

If $l_{0}=2, l_{1}=l_{2}=l_{3}=0$, then the values $\alpha$ and $\kappa$ are expressed as

$$
\wp(\alpha)=e_{1}-\frac{\left(E-3 e_{1}\right)\left(E+6 e_{1}\right)^{2}}{9\left(E^{2}-3 g_{2}\right)}, \quad \kappa=\frac{2}{3\left(E^{2}-3 g_{2}\right)} \sqrt{-Q(E)} .
$$

\subsection{Relationship among commuting operators}

We review a relationship among the operators $H, A$, the polynomial $Q(E)$ and the invariant subspaces. On the operators $H$ and $A$, we have the following relation: 
Proposition 11 ([44], Proposition 3.2). Let H and A be the operators defined by equation (2.4) and equations (2.2), (2.3), and $Q(E)$ be the polynomial defined in equation (2.10). Then

$$
A^{2}+Q(H)=0 \text {. }
$$

It is known that, if $l_{0}, l_{1}, l_{2}, l_{3} \in \mathbb{Z}_{\geq 0}$, then there exist four invariant subspaces with respect to the action of the operator $H$. We describe the spaces more precisely. Let $V_{\alpha_{0}, \alpha_{1}, \alpha_{2}, \alpha_{3}}$ be the space defined in Proposition 2 and

$$
U_{\alpha_{0}, \alpha_{1}, \alpha_{2}, \alpha_{3}}= \begin{cases}V_{\alpha_{0}, \alpha_{1}, \alpha_{2}, \alpha_{3}}, & \sum_{i=0}^{3} \alpha_{i} / 2 \in \mathbb{Z}_{\leq 0} ; \\ V_{1-\alpha_{0}, 1-\alpha_{1}, 1-\alpha_{2}, 1-\alpha_{3}}, & \sum_{i=0}^{3} \alpha_{i} / 2 \in \mathbb{Z}_{\geq 2} ; \\ \{0\}, & \text { otherwise. }\end{cases}
$$

If $l_{0}, l_{1}, l_{2}, l_{3} \in \mathbb{Z}_{\geq 0}$ and $l_{0}+l_{1}+l_{2}+l_{3}$ is even, then the operator $H$ preserves the space

$$
V=U_{-l_{0},-l_{1},-l_{2},-l_{3}} \oplus U_{-l_{0},-l_{1}, l_{2}+1, l_{3}+1} \oplus U_{-l_{0}, l_{1}+1,-l_{2}, l_{3}+1} \oplus U_{-l_{0}, l_{1}+1, l_{2}+1,-l_{3}},
$$

and also preserves the components in equation (2.15). If $l_{0}, l_{1}, l_{2}, l_{3} \in \mathbb{Z}_{\geq 0}$ and $l_{0}+l_{1}+l_{2}+l_{3}$ is odd, then the operator $H$ preserves the space

$$
V=U_{-l_{0},-l_{1},-l_{2}, l_{3}+1} \oplus U_{-l_{0},-l_{1}, l_{2}+1,-l_{3}} \oplus U_{-l_{0}, l_{1}+1,-l_{2},-l_{3}} \oplus U_{l_{0}+1,-l_{1},-l_{2},-l_{3}},
$$

and also preserves the components in equation (2.16). Then we have

Proposition 12 ([46]). (i) The operator $A$ annihilates any elements in the space $V$.

(ii) The monic characteristic polynomial of the space $V$ with respect to the action of $H$ coincides with $Q(E)$.

Note that the operator $A$ was constructed by composing the generalized Darboux transformations which are related to the spaces in the components of equation (2.15) or equation (2.16).

\section{Results on the Calogero-Moser-Sutherland model and the Inozemtsev model}

We are going to consider multidimensional generalizations of Lamé's equation and Heun's equation in the elliptic form. For this purpose, we introduce the quantum mechanical systems.

\subsection{The elliptic Calogero-Moser-Sutherland model}

The elliptic Calogero-Moser-Sutherland model (or the elliptic Olshanetsky-Perelomov model [31]) of type $A_{N-1}$ is a quantum many-body system whose Hamiltonian is given as follows:

$$
H=-\frac{1}{2} \sum_{i=1}^{N} \frac{\partial^{2}}{\partial x_{i}^{2}}+l(l+1) \sum_{1 \leq i<j \leq N} \wp\left(x_{i}-x_{j}\right),
$$

where $\wp(x)$ is the Weierstrass elliptic function. For the case $N=2$, the model reproduces Lamé's equation by setting $x_{1}-x_{2}=x$ and restricting to the line $x_{1}+x_{2}=0$.

This model is known to be completely integrable, i.e., there exist $N$-algebraically independent commuting operators $P_{k}(k=1, \ldots, N)$ which commute with the Hamiltonian $H$. Namely, by setting

$$
P_{k}=\sum_{0 \leq j \leq[k / 2]} \frac{(l(l+1))^{j}}{2^{j} j !(k-2 j) !} \sum_{\sigma \in S_{N}} \sigma\left(\wp\left(x_{1}-x_{2}\right) \wp\left(x_{3}-x_{4}\right) \cdots\right.
$$




$$
\left.\times \wp\left(x_{2 j-1}-x_{2 j}\right) \partial_{2 j+1} \partial_{2 j+2} \cdots \partial_{k}\right)
$$

where $S_{N}$ is the symmetric group, $[x]$ is the integral part of $x$ and $\partial_{i}=\partial / \partial x_{i}$, we have $\left[P_{k}, H\right]=0$ $(1 \leq k \leq N)$ and $\left[P_{k}, P_{k^{\prime}}\right]=0\left(1 \leq k, k^{\prime} \leq N\right)$ (see [30]). The Hamiltonian $H$ is expressed as $H=P_{2}-P_{1}^{2} / 2$.

By the trigonometric limit $(\tau \rightarrow \sqrt{-1} \infty)$ of the elliptic Calogero-Moser-Sutherland model where $(1, \tau)$ is the basic periods of the elliptic function, we obtain (up to an additive scalar) the Hamiltonian of the trigonometric Calogero-Moser-Sutherland model,

$$
H_{\text {trig }}=-\frac{1}{2} \sum_{i=1}^{N} \frac{\partial^{2}}{\partial x_{i}^{2}}+\pi^{2} l(l+1) \sum_{1 \leq i<j \leq N} \frac{1}{\sin ^{2}\left(\pi\left(x_{i}-x_{j}\right)\right)} .
$$

The eigenstates of the Calogero-Sutherland model are described by the Jack polynomial $J_{\lambda}^{\left(\frac{1}{l+1}\right)}(X)\left(\lambda \in \mathcal{M}_{N}\right)($ see $[39])$, i.e.,

$$
H_{\text {trig }}\left(J_{\lambda}^{\left(\frac{1}{l+1}\right)}(X) \Delta(X)^{l+1}\right)=\left(e_{0}+2 \pi^{2} E_{\lambda}^{\left[\frac{1}{l+1}\right]}\right) J_{\lambda}^{\left(\frac{1}{l+1}\right)}(X) \Delta(X)^{l+1},
$$

where $X_{i}=\exp \left(2 \pi \sqrt{-1} x_{i}\right), \mathcal{M}_{N}=\left\{\lambda=\left(\lambda_{1}, \lambda_{2}, \ldots, \lambda_{N}\right) \mid i>j \Rightarrow \lambda_{i}-\lambda_{j} \in \mathbb{Z}_{\geq 0}\right\}, e_{0}=$ $\frac{1}{6} \pi^{2}(l+1)^{2} N\left(N^{2}-1\right), \Delta(X)=\left(X_{1} X_{2} \cdots X_{N}\right)^{\frac{1-N}{2}} \prod_{i<j}\left(X_{i}-X_{j}\right)$ and $E_{\lambda}^{[\alpha]}=\sum_{i=1}^{N} \lambda_{i}^{2}+\sum_{i=1}^{N} \frac{N+1-2 i}{\alpha} \lambda_{i}$. In particular, the ground-state is given by $\Delta(X)^{l+1}$. Several properties of the Jack polynomial were studied. Vadim Kuznetsov and his collaborators studied the Jack polynomial and related polynomials from the aspects of separation of variable [25], the Pfaff lattice [1] and the $Q$ operator [24].

In contrast with the trigonometric models, the elliptic models are less investigated and the spectra or the eigenfunctions are not sufficiently analyzed. There is, however, some important progress due to Felder and Varchenko. They introduced the Bethe Ansatz method for the $N$ particle elliptic Calogero-Moser model with the coupling constant $l$ a positive integer. Note that Hermite essentially introduced the Bethe Ansatz method for the case $N=2$ and $l \in \mathbb{Z}$ (see [49]), and Dittrich and Inozemtsev [9] did it for the case $N=3$ and $l=1$ in a different representation.

Fix the parameters $N$ and $l$. We set $m=l N(N-1) / 2$. Let $c:\{1, \ldots, m\} \rightarrow\{1, \ldots, N\}$ be the unique non-decreasing function such that $c^{-1}(j)$ has $(N-j) l$ elements. Let $\epsilon_{i}(1 \leq i \leq N)$ be an orthonormal basis of $\mathbb{R}^{N}$ with an inner product $(\cdot, \cdot)$. Set $\alpha_{i}=\epsilon_{i}-\epsilon_{i+1}, \mathfrak{h}^{*}=\left\{\sum_{i=1}^{N} x_{i} \epsilon_{i} \mid \sum_{i=1}^{N} x_{i}=0\right\}$, $p_{i}=i(2 N-i-1) l / 2$ and $V_{i}=\left\{p_{i-1}+1, p_{i-1}+2, \ldots, p_{i}\right\}(1 \leq i \leq N-1)$. Let $W$ be the set of maps $w=\left(w_{1}, \ldots, w_{N}\right)\left(w_{i}: V_{i} \rightarrow\{i, i+1, \ldots, N-1\}\right)$ such that $\#\left\{w_{i}^{-1}(j)\right\}=l$ for $1 \leq i \leq j \leq N-1$. For $w=\left(w_{1}, \ldots, w_{N-1}\right) \in W$, let $F_{w}$ be the set of maps $f=\left(f_{1}, \ldots, f_{N-2}\right)$ $\left(f_{i}: V_{i+1} \rightarrow V_{i}\right.$ ) such that (i) $f_{i}$ is injective (ii) If $f_{i}(x)=y$ then $w_{i+1}(x)=w_{i}(y)$. Set

$$
\begin{aligned}
& \theta_{1}(x)=2 \sum_{n=1}^{\infty}(-1)^{n-1} \exp \left(\tau \pi \sqrt{-1}(n-1 / 2)^{2}\right) \sin (2 n-1) \pi x, \\
& \theta(x)=\frac{\theta_{1}(x)}{\theta_{1}^{\prime}(0)}, \quad \sigma_{\lambda}(x)=\frac{\theta^{\prime}(0) \theta(x-\lambda)}{\theta(x) \theta(\lambda)} .
\end{aligned}
$$

For $\xi \in \mathfrak{h}^{*}$, we introduce the functions $\Phi_{\tau}\left(t_{1}, \ldots, t_{m}\right)$ and $\omega(t ; x)$ as follows

$$
\begin{aligned}
\Phi_{\tau}\left(t_{1}, \ldots, t_{m}\right)= & e^{2 \pi \sqrt{-1}\left(\xi, \sum_{j} t_{j} \alpha_{c(j)}\right)} \\
& \times \prod_{1 \leq j \leq(N-1) l} \theta\left(t_{j}\right)^{-l N} \prod_{\substack{c(i)=c(j) \\
i<j}} \theta\left(t_{i}-t_{j}\right)^{2} \prod_{\substack{|c(i)-c(j)|=1 \\
i<j}} \theta\left(t_{i}-t_{j}\right)^{-1},
\end{aligned}
$$




$$
\omega(t ; x)=e^{2 \pi \sqrt{-1}\left(\xi, \sum_{i} x_{i} \epsilon_{i}\right)} \sum_{w \in W} \sum_{f \in F_{w}} \prod_{i=1}^{N-1} \prod_{k=p_{i-1}+1}^{p_{i}} \sigma_{x_{i}-x_{w_{i}(k)+1}}\left(t_{k}-t_{f_{i}(k)}\right),
$$

where $t_{0}=0, f_{0}(k)=0$. Then we have

Proposition $13([12,13,11])$. If $\left(t_{1}^{0}, \ldots, t_{m}^{0}\right)$ satisfy the following Bethe Ansatz equations,

$$
\left.\frac{\partial \Phi_{\tau}}{\partial t_{i}}\right|_{\left(t_{1}^{0}, \ldots, t_{m}^{0}\right)}=0 \quad(1 \leq i \leq m)
$$

the function $\omega\left(t^{0} ; x\right)$ is an eigenfunction of the Hamiltonian $H$ with the eigenvalue

$$
2 \pi^{2}(\xi, \xi)-2 \pi \sqrt{-1} \frac{\partial}{\partial \tau} S\left(t_{1}^{0}, \ldots, t_{m}^{0} ; \tau\right)-l(l+1)(N-1) N \eta,
$$

where

$$
\begin{aligned}
& S\left(t_{1}, \ldots, t_{m} ; \tau\right)=\sum_{i<j}\left(\alpha_{c(i)}, \alpha_{c(j)}\right) \log \theta\left(t_{i}-t_{j}\right)-\sum_{c(i)=1} l N \log \theta\left(t_{i}\right), \\
& \eta=\pi^{2}\left(\frac{1}{6}-4 \sum_{n=1}^{\infty} \frac{p^{n}}{1-p^{n}}\right) \quad \text { and } \quad p=\exp (2 \pi \sqrt{-1} \tau) .
\end{aligned}
$$

Therefore, if we find solutions to the Bethe Ansatz equations, we can investigate the CalogeroMoser-Sutherland model in more detail. There are two things to be considered for applying Proposition 13 to the spectral problem of the elliptic Calogero-Moser-Sutherland model. The first one is to find the condition when the eigenfunctions obtained by the Bethe Ansatz method are connected to square-integrable eigenstates and the second one is how the solutions of the Bethe Ansatz equation behave.

On the first question, the condition is described as the parameter $\xi$ belonging to some lattice (the weight lattice of type $A_{N-1}$ ). By symmetrizing or anti-symmetrizing the function $\omega\left(t^{0} ; x\right)$, we obtain square-integrable eigenstates, although we must check that they are identically zero or not.

On the second question, we consider the solution at $p=\exp (2 \pi \sqrt{-1} \tau)=0$ (the case of the trigonometric limit $\tau \rightarrow \sqrt{-1} \infty$ ) and look into the behavior where $p$ is near 0 , because it is hopeful to solve the Bethe Ansatz equations for the trigonometric case in contrast to being hopeless directly for the elliptic case. A key tool to connect the trigonomertic solutions to the elliptic solutions is the implicit function theorem. Thus we construct the square-integrable eigenstates and obtain the main result in [40], which gives a sufficient condition for regular convergence of the perturbation expansion. In particular, for the case $N=2, l \in \mathbb{Z}_{\geq 1}$ and the case $N=3, l=1$, we have convergence of the perturbation series for all eigenstates related to the Jack polynomial.

Note that this idea can be interpreted to consider the elliptic Calogero-Moser-Sutherland model by perturbation from the trigonometric Calogero-Moser-Sutherland model. Convergence for the general cases was proved in [23] by another method. Namely, by applying Kato-Rellich theory, we have convergence of the perturbation series in $p$ for $l \geq 0$ and arbitrary $N$. The eigenvalues and the eigenfunctions are calculated as power series by a standard algorithm of perturbation. Remark that Fernandez, Garcia and Perelomov [14] derived a fully explicit formula for second order in $p$, and Langmann $[27,28]$ obtained another algorithm for constructing the eigenfunctions and the eigenvalues as formal power series of $p$, which also gives a formula for all orders in $p$.

On the Bethe Ansatz for the elliptic Calogero-Moser-Sutherland model, there are some problems to be solved. For example, it has not been shown at the moment of writing that the eigenfunction $\omega\left(t^{0}, x\right)$ written in the form of the Bethe Ansatz is also an eigenfunction of the higher commuting operators $P_{3}, \ldots, P_{N}$ (see also [35]). 


\subsection{The Inozemtsev model}

We now introduce a quantum mechanical system that is a multidimensional generalization of Heun's equation in the elliptic form.

The Inozemtsev model of type $B C_{N}$ [20] is a quantum mechanical system with $N$-particles whose Hamiltonian is given by

$$
\begin{aligned}
H= & -\sum_{j=1}^{N} \frac{\partial^{2}}{\partial x_{j}^{2}}+2 l(l+1) \sum_{1 \leq j<k \leq N}\left(\wp\left(x_{j}-x_{k}\right)+\wp\left(x_{j}+x_{k}\right)\right) \\
& +\sum_{j=1}^{N} \sum_{i=0}^{3} l_{i}\left(l_{i}+1\right) \wp\left(x_{j}+\omega_{i}\right),
\end{aligned}
$$

where $l$ and $l_{i}(i=0,1,2,3)$ are coupling constants. This is also a generalization of the elliptic Calogero-Moser-Sutherland model of type $B C_{N}$.

The Inozemtsev model of type $B C_{N}$ is completely integrable, i.e., there exist $N$ algebraically independent mutually commuting differential operators $P_{k}(k=1, \ldots, N)$ (higher commuting Hamiltonians) which commute with the Hamiltonian of the model, and Oshima [32] described the commuting operators explicitly. Note that the Inozemtsev model of type $B C_{N}$ (resp. the elliptic Calogero-Moser-Sutherland model of type $A_{N}$ ) is a universal completely integrable model of quantum mechanics with the symmetry of the Weyl group of type $B_{N}$ (resp. type $A_{N}$ ), which follows from the classification due to Ochiai, Oshima and Sekiguchi [30, 33]. For the case $N=1$, the operator (3.2) appears in the elliptic form of Heun's equation (2.4). Therefore the Inozemtsev model of type $B C_{N}$ is regarded as a multidimensional generalization of Heun's equation.

On the trigonometric limit $\tau \rightarrow \sqrt{-1} \infty$, we obtain the trigonometric Calogero-MoserSutherland model of type $B C_{N}$, and we can investigate the Inozemtsev model of type $B C_{N}$ by perturbation from the trigonometric model [23, 47].

A method of quasi-solvability is available on the Inozemtsev model of type $B C_{N}$. Finkel et al. studied quasi-solvable models in $[15,16]$, and they found several quasi-exactly solvable many-body systems including the Inozemtsev model of type $B C_{N}$. We now describe the finitedimensional spaces which are related to the quasi-solvability. The quasi-solvability with respect to the Hamiltonian $H$ was established in [16] and reformulated in [41].

Proposition $14([16,41])$. Let $a, b_{i}(i=0,1,2,3)$ be the numbers which satisfy $a \in\{-l, l+1\}$ and $b_{i} \in\left\{-l_{i} / 2,\left(l_{i}+1\right) / 2\right\}(i=0,1,2,3)$. Set

$$
\Phi(z)=\prod_{1 \leq j<k \leq N}\left(z_{j}-z_{k}\right)^{a} \prod_{j=1}^{N} \prod_{i=1}^{3}\left(z_{j}-e_{i}\right)^{b_{i}} .
$$

Assume that $d=-\left((N-1) a+b_{0}+b_{1}+b_{2}+b_{3}\right)$ is a non-negative integer. Let $W_{d}^{\mathrm{sym}}$ be the space spanned by

$$
\Phi\left(\wp\left(x_{1}\right), \wp\left(x_{2}\right), \ldots, \wp\left(x_{N}\right)\right) \sum_{\sigma \in S_{N}} \wp\left(x_{1}\right)^{m_{\sigma(1)}} \wp\left(x_{2}\right)^{m_{\sigma(2)}} \cdots \wp\left(x_{N}\right)^{m_{\sigma(N)}}
$$

such that $m_{i} \in\{0,1, \ldots, d\}$ for all $i$. Then we have

$$
H \cdot W_{d}^{\mathrm{sym}} \subset W_{d}^{\mathrm{sym}}
$$

The quasi-solvability was extended to the commuting differential operators. 
Proposition 15 ([41], Theorem 3.3). Assume that $d=-\left((N-1) a+b_{0}+b_{1}+b_{2}+b_{3}\right)$ is a non-negative integer. Then $P_{k} \cdot W_{d}^{\mathrm{sym}} \subset W_{d}^{\mathrm{sym}}$ for $k=1,2, \ldots, N$, where $W_{d}^{\mathrm{sym}}$ is the finitedimensional space defined in Proposition 14 and $P_{k}$ are the commuting differential operators which ensure the complete integrability.

By the quasi-solvability, finitely-many eigenvalues and eigenfunctions are calculated by diagonalizing the commuting matrices simultaneously for the case that the assumption of Proposition 14 is true. The eigenfunctions obtained by the quasi-solvability may not be square-integrable in general, although the eigenfunctions for the case that the parameters $a, b_{0}, b_{1}$ in Proposition 14 are chosen as $a=l+1, b_{0}=\left(l_{0}+1\right) / 2$ and $b_{1}=\left(l_{1}+1\right) / 2$ are square-integrable.

It seems that an explicit expression of the Bethe Ansatz as Proposition 13 for the Inozemtsev model of type $B C_{N}$ and corresponding conformal field theory are not known in the moment of writing, although Chalykh, Etingof and Oblomkov [6] gave a general recipe for calculating the Bloch eigenfunctions. They showed that these are parametrized by a certain algebraic variety (the Hermite-Bloch variety) which can be computed. This would lead to a version of the Bethe Ansatz for the models including the Inozemtsev model of type $B C_{N}$, though these Bethe Ansatz equations would be rather complicated. For a special case of the $B C_{2}$ case, this scheme is worked out explicitly in $[6, \S 6]$.

We hope to investigate the Bethe Ansatz for the Inozemtsev model of type $B C_{N}$ to study the model in more detail.

\section{Towards finite-gap integration of the Inozemtsev model}

In Section 2, we reviewed the finite-gap integration of Heun's equation, and observed that the existence of commuting operator of odd order plays important roles.

For a multidimensional generalization of finite-gap integration, Chalykh and Veselov introduced the notion "algebraic integrability". The Schrödinger operator $L=-\sum_{i=1}^{N} \partial^{2} / \partial x_{i}^{2}+$ $u\left(x_{1}, \ldots, x_{N}\right)$ is called completely integrable [8], if there exist $N$ commuting operators $L_{1}=$ $L, L_{2}, \ldots, L_{N}$ with algebraically independent constant highest symbols $s_{1}(\xi)\left(=\xi_{1}^{2}+\cdots+\xi_{N}^{2}\right)$, $s_{2}(\xi), \ldots, s_{N}(\xi)\left(\xi_{j}=\sqrt{-1} \partial / \partial x_{j}\right)$. For example, the Calogero-Moser-Sutherland models are completely integrable. On the model of type $A_{N}$, the highest symbols of $P_{k}$ (see equation (3.1)) are written as $\left((-\sqrt{-1})^{k} / k !\right) \sum_{i_{1}<i_{2}<\cdots<i_{k}} \xi_{i_{1}} \xi_{i_{2}} \cdots \xi_{i_{k}}$. The Inozemtsev model of type $B C_{N}$ is also completely integrable. The operator $L$ is called algebraically integrable in the sense of [8], if $L$ is completely integrable and there exists one more operator $L_{0}$ commuting with $L_{i}(i=1, \ldots, N)$ and the highest symbol $s_{0}(\xi)$ of $L_{0}$ takes the distinct values at the roots of the equations $s_{i}(\xi)=E_{i}(i=1, \ldots, N)$ for almost all $E_{i}$.

On Heun's equation in the elliptic form, if $l_{0}, l_{1}, l_{2}, l_{3}$ are integers, then it is algebraically integrable, because there exists a commuting operator $A$ of odd order. Thus we expect that algebraically integrable Schrödinger operators also have rich properties.

Chalykh and Veselov conjectured [7] that the Calogero-Moser-Sutherland model with integral coupling constants are algebraically integrable. For the case of type $A_{N}$, Braverman, Etingof and Gaitsgory [4] obtained algebraic integrability. More precisely, they established that, if $l$ is a positive integer, then the operator

$$
H=-\frac{1}{2} \sum_{i=1}^{N} \frac{\partial^{2}}{\partial x_{i}^{2}}+l(l+1) \sum_{1 \leq i<j \leq N} \wp\left(x_{i}-x_{j}\right)
$$

is algebraically integrable by applying the Bethe Ansatz due to Felder-Varchenko (see Section 3.1) and the differential Galois theory. In [6], Chalykh, Etingof and Oblomkov proved 
that the Chalykh-Veselov conjecture is true and the Inozemtsev model of type $B C_{N}$ (see equation (3.2)) is also algebraically integrable, if $l, l_{0}, l_{1}, l_{2}, l_{3}$ are all integers. Their method relies on results on the differential Galois theory obtained in [4] and the local triviality of the monodromy. On an application of the algebraic integrability, the eigenfunctions of the Baker-Akhiezer (Bloch) type are considered. We expect further studies for applications of the algebraic integrability on the Calogero-Moser-Sutherland models and the Inozemtsev models.

The explicit expressions of the extra commuting operators were obtained and investigated by Oblomkov, Khodarinova and Prikhodsky [29, 21, 22] for the case $l=1$ on the Calogero-MoserSutherland model of type $A_{3}$ and the case $l=l_{0}=1, l_{1}=l_{2}=l_{3}=0$ on the Inozemtsev model of type $\mathrm{BC}_{2}$. On the Calogero-Moser-Sutherland model of type $A_{3}$ with $l=1$, the Hamiltonian and commuting operators which guarantee the complete integrability are given as

$$
\begin{aligned}
& H=-\left(\partial_{1}^{2}+\partial_{2}^{2}+\partial_{3}^{2}\right) / 2+2\left(\wp_{12}+\wp_{23}+\wp_{31}\right), \\
& P_{1}=\partial_{1}+\partial_{2}+\partial_{3}, \\
& P_{3}=\partial_{1} \partial_{2} \partial_{3}+2 \wp_{12} \partial_{3}+2 \wp_{23} \partial_{1}+2 \wp_{31} \partial_{2},
\end{aligned}
$$

(see equation (3.1)) where we have used the notations $\partial_{i}=\partial / \partial x_{i}$ and $\wp_{i j}=\wp\left(x_{i}-x_{j}\right)$. The additional commuting operators are written as

$$
\begin{aligned}
I_{12}= & \left(\partial_{1}-\partial_{3}\right)^{2}\left(\partial_{2}-\partial_{3}\right)^{2}-8 \wp_{23}\left(\partial_{1}-\partial_{3}\right)^{2}-8 \wp_{13}\left(\partial_{2}-\partial_{3}\right)^{2} \\
& +4\left(\wp_{12}-\wp_{13}-\wp_{23}\right)\left(\partial_{1}-\partial_{3}\right)\left(\partial_{2}-\partial_{3}\right)-2\left(\wp_{12}^{\prime}+\wp_{13}^{\prime}+6 \wp_{23}^{\prime}\right)\left(\partial_{1}-\partial_{3}\right) \\
& -2\left(-\wp_{12}^{\prime}+6 \wp_{13}^{\prime}+\wp_{23}^{\prime}\right)\left(\partial_{2}-\partial_{3}\right)-2 \wp_{12}^{\prime \prime}-6 \wp_{13}^{\prime \prime}-6 \wp_{23}^{\prime \prime}+4\left(\wp_{12}^{2}+\wp_{13}^{2}+\wp_{23}^{2}\right) \\
& +8\left(\wp_{12} \wp_{13}+\wp_{12} \wp_{23}+7 \wp_{13} \wp_{23}\right),
\end{aligned}
$$

$I_{23}$ and $I_{31}$, which are written by permuting the indices. Then any non-symmetric linear combination of them, e.g., $L_{4}=I_{12}+2 I_{23}$ would fit into the definition of algebraic integrability (see [21]). Explicit expressions of the extra commuting operators for the models which have symmetry of the deformed root system $A_{3}(m)$ or $B_{2}(l, m)$ were also obtained.

Another possible method for constructing extra commuting operators is the multidimensional Darboux transformation, because the commuting operator for the case of Heun's equation is constructed by composing the (generalized) Darboux transformations. Multidimensional Darboux transformations were studied from several viewpoints $[2,18,36,5]$. In [2], based on the existence of an explicit eigenfunction of the Hamiltonian $H\left(=H^{(0)}\right)$ with a certain eigenvalue, an alternate Hamiltonian $\tilde{H}\left(=H^{(N)}\right)$, matrix valued operators $H^{(i)}(i=1, \ldots, N-1)$ and supersymmetry operators $Q_{j+1, j}^{-}$and $Q_{j, j+1}^{+}(j=0, \ldots, N-1)$ which connect $H^{(j)}$ and $H^{(j+1)}$ were introduced and studied. On the other hand, we know an explicit eigenfunction of the Inozemtsev model of type $B C_{N}$, if the value $d\left(=-\left((N-1) a+b_{0}+b_{1}+b_{2}+b_{3}\right)\right)\left(a \in\{-l, l+1\}, b_{i} \in\left\{-l_{i} / 2,\left(l_{i}+1\right) / 2\right\}\right.$ $(i=0,1,2,3))$ in the assumption of Proposition 14 is equal to zero. Then the alternate Hamiltonian $\tilde{H}$ with respect to $H$ in equation (3.2) would be written as

$$
\begin{aligned}
\tilde{H}= & -\sum_{j=1}^{N} \frac{\partial^{2}}{\partial x_{j}^{2}}+2 a(a+1) \sum_{j<k}\left(\wp\left(x_{j}-x_{k}\right)+\wp\left(x_{j}+x_{k}\right)\right) \\
& +\sum_{j=1}^{N} \sum_{i=0}^{3} 2 b_{i}\left(2 b_{i}+1\right) \wp\left(x_{j}+\omega_{i}\right) .
\end{aligned}
$$

In the moment of writing, we do not know an operator $L$ which directly intertwines the operators $H$ and $\tilde{H}$ as $\tilde{H} L=L H$. We expect applications of the multidimensional Darboux transformation for the analysis of the elliptic Calogero-Moser-Sutherland model or the Inozemtsev model. 


\section{Acknowledgements}

The author would like to thank the referees for valuable comments.

\section{References}

[1] Adler M., Kuznetsov V.B., van Moerbeke P., Rational solutions to the Pfaff lattice and Jack polynomials, Ergodic Theory Dynam. Systems 22 (2002), 1365-1405, nlin.SI/0202037.

[2] Andrianov A.A., Borisov N.V., Ioffe M.V., The factorization method and the Darboux transformation for multidimensional Hamiltonians, Theoret. and Math. Phys. 61 (1984), 1078-1088.

[3] Aoyama H., Sato M., Tanaka T., $\mathcal{N}$-fold supersymmetry in quantum mechanics: general formalism, Nuclear Phys. B 619 (2001), 105-127, quant-ph/0106037.

[4] Braverman A., Etingof P., Gaitsgory D., Quantum integrable systems and differential Galois theory, Transfor. Groups 2 (1997), 31-57, alg-geom/9607012.

[5] Chalykh O.A., Darboux transformations for multidimensional Schrödinger operators, Russian Math. Surveys 53 (1998), no. 2, 167-168.

[6] Chalykh O.A., Etingof P., Oblomkov A., Generalized Lamé operators, Comm. Math. Phys. 239 (2003), 115-153, math.QA/0212029.

[7] Chalykh O.A., Veselov A.P., Commutative rings of partial differential operators and Lie algebras, Comm. Math. Phys. 126 (1990), 597-611.

[8] Chalykh O.A., Veselov A.P., Integrability in the theory of Schrödinger operator and harmonic analysis, Comm. Math. Phys. 152 (1993), 29-40.

[9] Dittrich J., Inozemtsev V.I., On the structure of eigenvectors of the multidimensional Lamé operator, J. Phys. A: Math. Gen. 26 (1993), L753-L756.

[10] Dubrovin B.A., Matveev V.B., Novikov S.P., Nonlinear equations of Korteweg-de Vries type, finite-band linear operators and Abelian varieties, Russian Math. Surveys 31 (1976), 59-146.

[11] Felder G., Rimanyi R., Varchenko A., Poincare-Birkhoff-Witt expansions of the canonical elliptic differential form, math.RT/0502296.

[12] Felder G., Varchenko A., Integral representation of solutions of the elliptic Knizhnik-ZamolodchikovBernard equations, Int. Math. Res. Not. (1995), no. 5, 221-233, hep-th/9502165.

[13] Felder G., Varchenko A., Three formulae for eigenfunctions of integrable Schrödinger operator, Compos. Math. 107 (1997), 143-175, hep-th/9511120.

[14] Fernandez N.J., Garcia F.W., Perelomov A.M., A perturbative approach to the quantum elliptic CalogeroSutherland model, Phys. Lett. A 307 (2003), 233-238, math-ph/0205042.

[15] Finkel F., Gomez-Ullate D., Gonzalez-Lopez A., Rodriguez M.A., Zhdanov R., $A_{N}$-type Dunkl operators and new spin Calogero-Sutherland models, Comm. Math. Phys. 221 (2001), 477-497, hep-th/0102039.

[16] Finkel F., Gomez-Ullate D., Gonzalez-Lopez A., Rodriguez M.A., Zhdanov R., New spin Calogero-Sutherland models related to $B_{N}$-type Dunkl operators, Nuclear Phys. B 613 (2001), 472-496, hep-th/0103190.

[17] Gesztesy F., Weikard R., Treibich-Verdier potentials and the stationary (m)KdV hierarchy, Math. Z. 219 (1995), 451-476.

[18] Gonzalez-Lopez A., Kamran N., The multidimensional Darboux transformation, J. Geom. Phys. 26 (1998), 202-226, hep-th/9612100.

[19] Ince E.L., Further investigations into the periodic Lamé functions, Proc. Roy. Soc. Edinburgh 60 (1940), 83-99.

[20] Inozemtsev V.I., Lax representation with spectral parameter on a torus for integrable particle systems, Lett. Math. Phys. 17 (1989), 11-17.

[21] Khodarinova L.A., Prikhodsky I.A., Algebraic spectral relations for elliptic quantum Calogero-Moser problems, J. Nonlinear Math. Phys. 6 (1999), 263-268, math-ph/0406050.

[22] Khodarinova L.A., Prikhodsky I.A., On algebraic integrability of the deformed elliptic Calogero-Moser problem, J. Nonlinear Math. Phys. 8 (2001), 50-53, math-ph/0406052.

[23] Komori Y., Takemura K., The perturbation of the quantum Calogero-Moser-Sutherland system and related results, Comm. Math. Phys. 227 (2002), 93-118, math.QA/0009244. 
[24] Kuznetsov V.B., Mangazeev V.V., Sklyanin, E.K., Q-operator and factorised separation chain for Jack polynomials, Indag. Math. (N.S.) 14 (2003), 451-482, math.CA/0306242.

[25] Kuznetsov V.B., Nijhoff F.W., Sklyanin E.K., Separation of variables for the Ruijsenaars system, Comm. Math. Phys. 189 (1997), 855-877, solv-int/9701004.

[26] Kuznetsov V.B., Sklyanin E.K., Separation of variables and integral relations for special functions, Ramanujan J. 3 (1999), 5-35, q-alg/9705006.

[27] Langmann E., Anyons and the elliptic Calogero-Sutherland model, Lett. Math. Phys. 54 (2000), 279-289, math-ph/0007036.

[28] Langmann E., A method to derive explicit formulas for an elliptic generalization of the Jack polynomials, in Jack, Hall-Littlewood and Macdonald Polynomials, Editors V.B. Kuznetsov and S. Sahi, Contemp. Math. 417 (2006), 257-270, math-ph/0511015.

[29] Oblomkov A.A., Integrability of some quantum systems associated with the root system $B_{2}$, Moscow Univ. Math. Bull. 54 (1999), 5-8.

[30] Ochiai H., Oshima T., Sekiguchi H., Commuting families of symmetric differential operators, Proc. Japan. Acad. 70 (1994), 62-66.

[31] Olshanetsky M.A., Perelomov A.M., Quantum integrable systems related to Lie algebras, Phys. Rep. 94 (1983), 313-404.

[32] Oshima T., Completely integrable systems with a symmetry in coordinates, Asian J. Math. 2 (1998), 935955.

[33] Oshima T., Sekiguchi H., Commuting families of differential operators invariant under the action of a Weyl group, J. Math. Sci. Univ. Tokyo 2 (1995), 1-75.

[34] Ronveaux A. (Editor), Heun's differential equations, Oxford University Press, Oxford, 1995.

[35] Ruijsenaars S.N.M., Elliptic integrable systems of Calogero-Moser type: a survey, in the Proceedings of Workshop on Elliptic Integrable Systems (2004, Kyoto), 201-221 (Notes by Y. Komori).

[36] Sabatier P.C., On multidimensional Darboux transformations, Inverse Problems 14 (1998), 355-366.

[37] Slavyanov S., Lay W., Special functions, Oxford University Press, Oxford, 2000.

[38] Smirnov A.O., Elliptic solitons and Heun's equation, in The Kowalevski Property, CRM Proc. Lecture Notes, Vol. 32, Amer. Math. Soc., Providence, 2002, 287-305, math.CA/0109149.

[39] Stanley R., Some combinatorial properties of Jack symmetric functions, Adv. Math. 77 (1989), 76-115.

[40] Takemura K., On the eigenstates of the elliptic Calogero-Moser model, Lett. Math. Phys. 53 (2000), 181-194, math.QA/0002104.

[41] Takemura K., Quasi-exact solvability of Inozemtsev models, J. Phys. A: Math. Gen. 35 (2002), 8867-8881, math.QA/0205274.

[42] Takemura K., The Heun equation and the Calogero-Moser-Sutherland system. I. The Bethe Ansatz method, Comm. Math. Phys. 235 (2003), 467-494, math.CA/0103077.

[43] Takemura K., The Heun equation and the Calogero-Moser-Sutherland system. II. The perturbation and the algebraic solution, Electron. J. Differential Equations (2004), no. 15, 30 pages, math.CA/0112179.

[44] Takemura K., The Heun equation and the Calogero-Moser-Sutherland system. III. The finite gap property and the monodromy, J. Nonlinear Math. Phys. 11 (2004), 21-46, math.CA/0201208.

[45] Takemura K., The Heun equation and the Calogero-Moser-Sutherland system. IV. The Hermite-Krichever Ansatz, Comm. Math. Phys. 258 (2005), 367-403, math.CA/0406141.

[46] Takemura K., The Heun equation and the Calogero-Moser-Sutherland system. V. The generalized Darboux transformations, J. Nonlinear Math. Phys. 13 (2006), 584-611, math.CA/0508093.

[47] Takemura K., Heun equation and Inozemtsev models, in Proceedings of The 24th Int. Coll. Group Theoretical Methods in Physics (July 15-20, 2002, Paris), Inst. Phys. Conf. Ser. 173 (2003), 605-608, nlin.SI/0303005.

[48] Treibich A., Verdier J.-L., Revetements exceptionnels et sommes de 4 nombres triangulaires, Duke Math. J. 68 (1992), 217-236.

[49] Whittaker E.T., Watson G.N., A course of modern analysis, 4th ed., Cambridge University Press, New York, 1962 . 